\title{
Cross Cultural Differences in Cognition: A Study on How Culture Affects the Way We Think
}

\author{
Sharon Campbell-Phillips ${ }^{1}$, Deb Proshad Halder ${ }^{2}$, Serlange Campbell ${ }^{3}$, Daneil \\ Phillips ${ }^{4}$ \\ ${ }^{1}$ Department of Education, University of the People, Pasadena, CA, USA \\ ${ }^{2}$ Lecturer, English, Jashore Government Girls' College, Bangladesh \\ ${ }^{3}$ Department of Nursing, College of Science, Technology and Applied Arts of Trinidad and Tobago \\ (COSTAATT) \\ ${ }^{4}$ Department of Business Management, University of the Southern Caribbean \\ beautifulblushes@yahoo.com
}

\section{Abstract}

Communication is the exchanging of information through speaking, writing and signals. It plays an important to our development; it is the dissemination of ideas, and information to persons. Cognition is our mental process in which we acquire knowledge and understanding, and this is done through our thoughts, our experiences, and our senses. Cultural differences involve what people' believe how they behave, the language they speak, and their practices based on their ethnicity. Cross-cultural differences in cognition can be very effective to certain operations conducted by persons; however, it can also limit us based on our perspective. To gather information and to understand how culture affects cognition and the way we think, questionnaires, surveys and experiments were used. Questionnaires were administered to tertiary level students, surveys were administered to teachers and experiments were conducted among students from various culture and background. The experiments were centered on visualization, focus and critical thinking. The purpose of this study is to investigate if cultural differences affect the way we think, and this double-dissociation is discussed in terms of implications for different developmental trajectories, with different developmental sub-tasks in the different cultures.
Keywords

cross culture; differences, cognition; learning

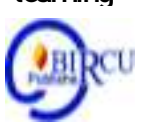

\section{Introduction}

Have you ever thought about the importance of cognition, and the role in which it plays in our lives? According to dictionary.com, cognition is the mental process which involves how we learn and understanding things. However, the ways in which we perceive and interpret thing are partly a result of cultural influences. In Plato's study of the mind, it suggests that people understand by firstly identifying the basic principles that are buried deep inside of them, which means that the knowledge people's gain from their culture will determine the way in which they learn. Within the communities and the world at large, there are many different cultures that are responsible for the way we think and behave. Aristotle, B.F. Skinner and John Locke also believed that people acquire their knowledge through their observations of the world around them. As stated in the articles, cognition differentiates across cultures, therefore, there are many different outcomes to how persons understand and express their views. Throughout development, the structure and function of the brain are influenced by the environment which is influenced by culture. 
Culture also affects the things people see, the way in which they are seen and people's' preferences. Cross-cultural differences are related to the different backgrounds, values, and beliefs of each culture. Therefore, because of the differences within cultures, there can be misunderstanding among persons who spend time working together. A study was conducted and it was discovered that even when people are presented with the same information at the same time, the information is processed differently because of cultural differences (Freeman et al., 2009). Another study that was done revealed that people are accurate at mind-reading members of their own culture (Adams et al., 2010). According to who persons affiliate themselves with, culture-brain interactions among persons can create confusion whether it is personal or political (Rule et al., 2010). Due to cultural differences, it is sometimes difficult to understand people from within other cultures, and this can create misunderstandings and conflicts among individuals. However, when persons are exposed to other cultures, they have the opportunity to learn and understand other cultures and the brain can become more culturally tuned, and this is very critical as it can affect our relationships, our career and our health.

\section{Review of Literature}

\subsection{Summary}

Despite our ethnicity, age, belief and cultural background, we all need depend on our cognitive process in order to develop and function properly in our different stages of life. Throughout history, culture has been an influence on the way people think and behave and learn. It is obvious that cultural differences influence people's cognitive process, however, despite the way people perceive things are according to culture and there are many differences, there are also some similarities and the way in which information is being processed seems to be the same among cultures. According to Vygotsky, the human cognition develops in a unique cultural context, which is the accumulated pattern of symbolic and non-symbolic tool that is used throughout historical existence. (Whorf, 1956) also contends that the particular language people of different culture speak affects their thought processes. In relation to the articles, cultural differences in cognition are seen even in both children and adults resulting in some cultures being independent and others interdependent. As a result of cultural differences, communication is broken down to a great extent, relationships are affected negatively, social interaction becomes almost impossible, work productivity is unsatisfactory and resolving issues and reasoning are unachievable. Therefore, it is critical to learn and understand the different cultures so that cultural barriers can be eliminated to some extent.

\section{Methodology}

For the purpose of this study, the system view approach was used to gather information. This approach allows flexibility in interaction. Experiments were conducted among tertiary students within different cultures. The aim is to collect primary data and this was done by using visual experiments. They were given various objects rich in colour to focus on and respond to a few questions, and then their responses were evaluated to see if their answers were influenced by their culture. This is critical as children's perspective should be evaluated and assessed based on social interaction with other persons and things. 
IV. Findings and Discussion

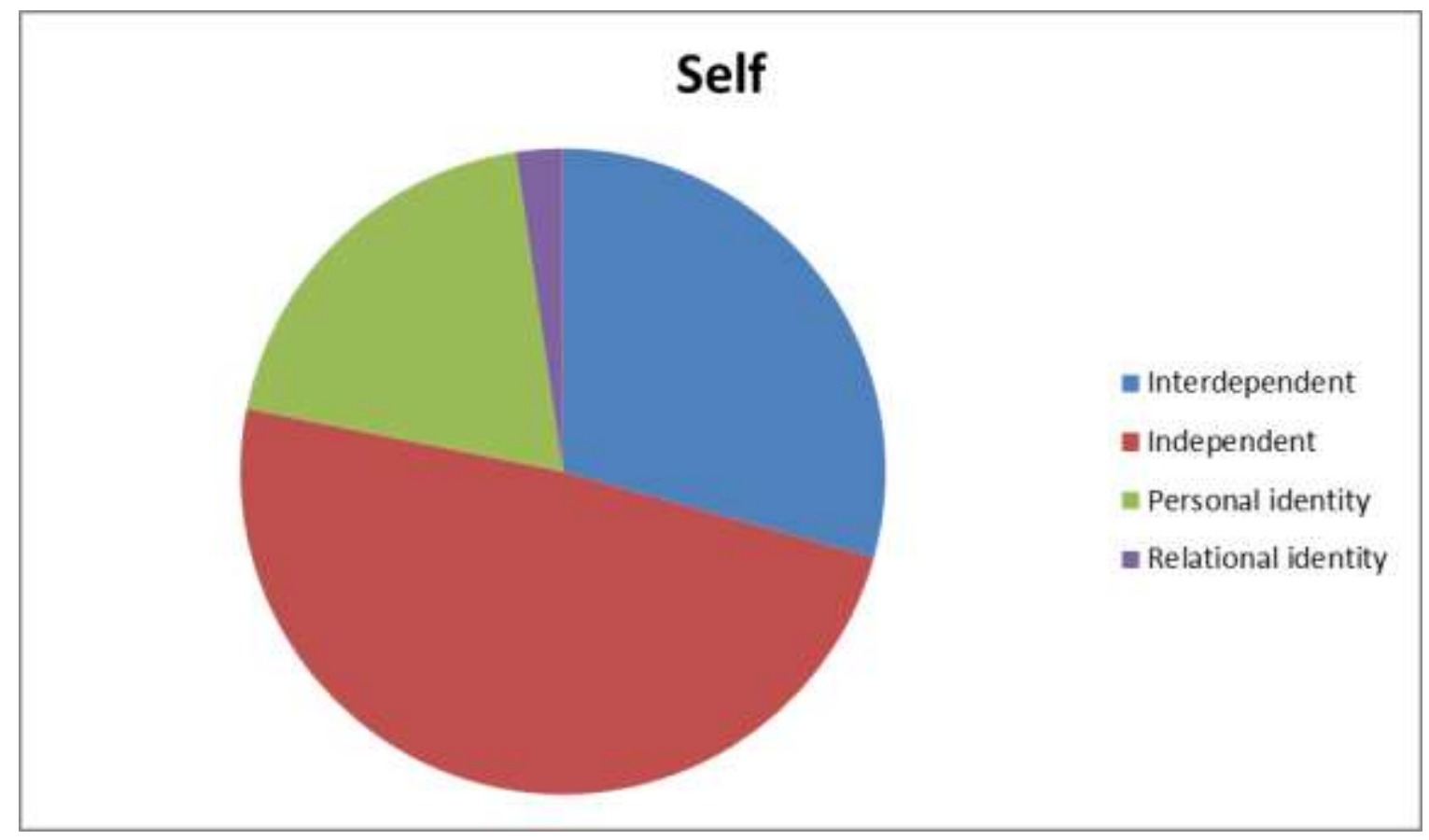

\section{Emotions}

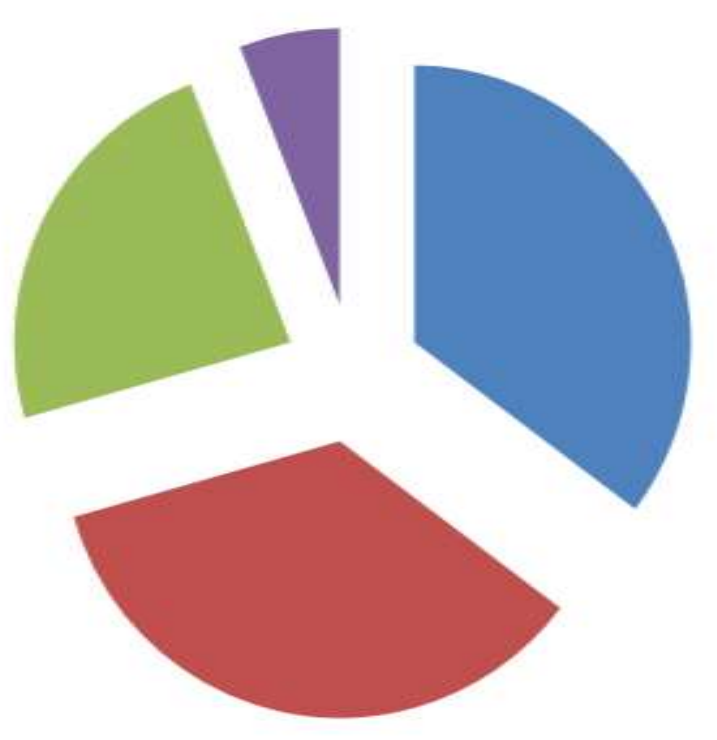

Engaging emotions

n Disengaging emotions

- happiness

- Sadness 


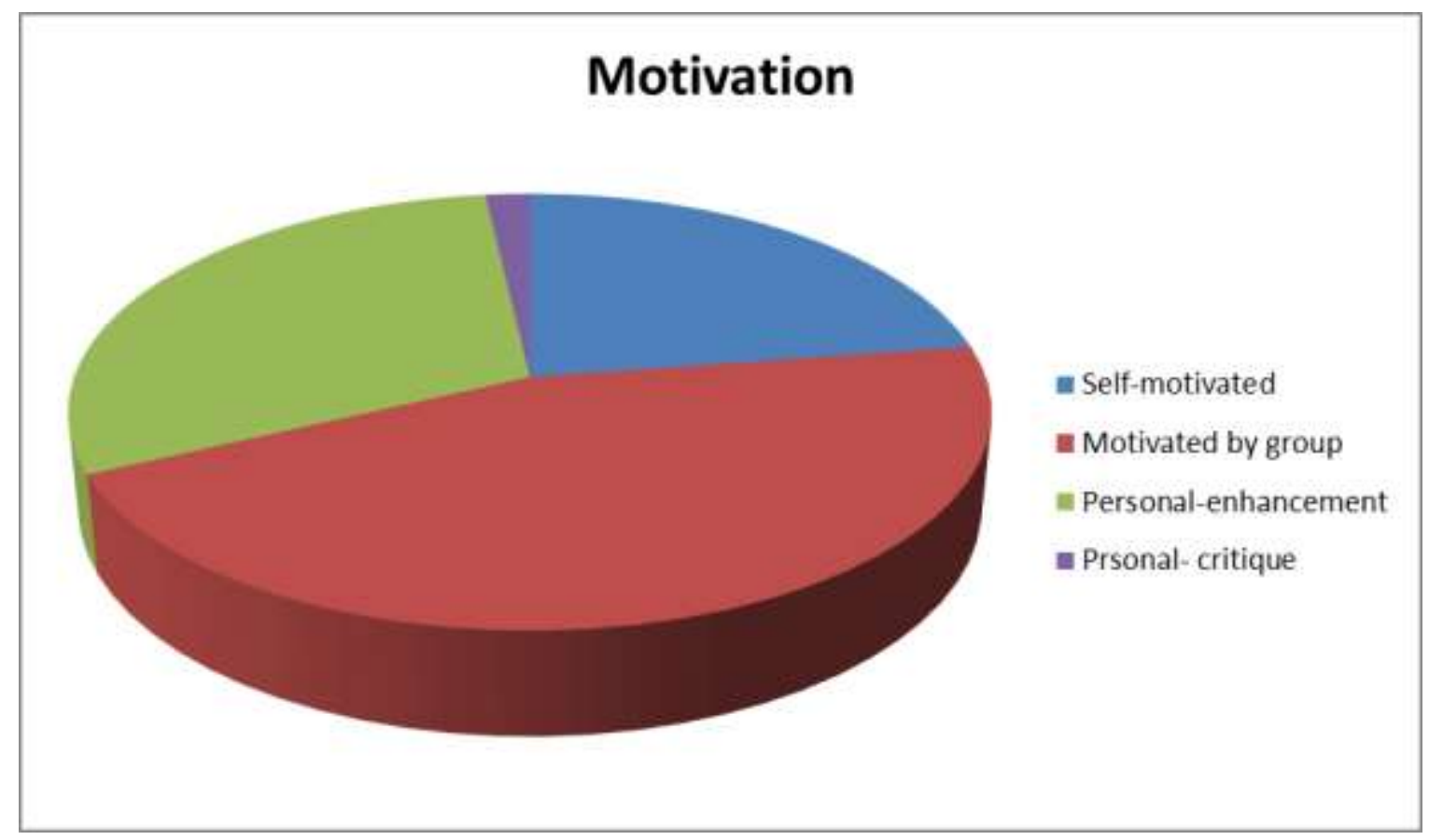

Though prior theories indicate that development is more or less universal across cultures, there is growing evidence of cross-cultural differences in adults (Chiao \& Blizinsky, 2010; Kim et al., 2010). In fact, Masuda \& Nisbett, 2001, 2006; Nisbett, 2003; Nisbett \& Miyamoto, 2005; Nisbett et al., 2001 found that Western and Eastern cultures appear to differ in the degree to which they emphasize decontextualized versus contextualized interpretations of objects; where westerners exhibited objected focused attention whereas Easterners exhibited more relational focused attention. Western and Eastern cultures appear to differ in the degree to which they emphasize decontextualized versus contextualized interpretations of objects (Masuda \& Nisbett, 2001, 2006; Nisbett, 2003; Nisbett \& Miyamoto, 2005; Nisbett et al., 2001). The key results primarily on studies of Western children but using a variety of experimental tasks suggest an early emphasis on objects and their individual properties and sensitivity to relational structure that emerges and becomes more robust during the late preschool period. It has been hypothesized that there is a shift during the preschool years from more object-based comparisons to more relational comparisons.

In experiment one, relational match-to-standard task in two conditions, with simple or richly detailed objects, Japanese children were generally better than U.S. children in making relational matches with both the simple and complex objects. In experiment 2 however, in the visual search task U.S. children outperformed their Japanese counterparts. Current finding indicates that there exists a bias for object-centered comparison by Western children and for more relational-centered comparisons by Eastern children. Third, the current results show, for the first time, an advantage for Western preschool children in visual search tasks; Western children found the target in a scene more rapidly than did Eastern children, suggesting that they were better able to maintain a focused search of the target object. Fourth, the results suggest that the relational structure in coherent scenes slows object search times for Japanese children relative to U.S. children. Gentner (1982) to proposed that relations and objects compete, such that children show more advanced relational reasoning when objects are abstract and simple and show less advanced relational reasoning when objects are rich and detailed. Overall, then, the pattern suggests a cross-cultural double dissociation in relational-focused versus object-focused tasks. 
Researchers have found that there is a cross culture difference in cognition when it comes to encoding objects in imagined social context. A study was done on East Asians participants and American participants and the researchers found that Japanese participants remembered more background information and their memory for objects was more impacted by the change in the background, in comparison to American participants (Lixia Yang, 2018). In another study, which was an eye tracking study to test the encoding of objects, Chua and colleagues noticed that American individuals focus more on objects whereas Chinese individuals demonstrated eye movements to their background (Lixia Yang, 2018).

In addition to this, East Asian researchers attended to study the field where they integrated targets and background comparison to Canadian participants. In terms of cognition differences amongst East Asians and Canadian, researchers revealed that East Asians may be more engaged in processing context when it is required to make meaningful item context association which helps their memory for contextual information (Lixia Yang, 2018). Yang and colleagues examined cultural differences and age related failure in memory for objects and their imagined social encoding contexts using an intentional context memory paradigm (Lixia Yang, 2018).

In the study, context was defined as an imagined social scenario in which stimuli were presented to participants for their evaluation (Lixia Yang, 2018). The study seeks to present the results of the difference in cognition of East Asian and Canadian. The research question seeks to find out "Do Chinese and Canadian participants differ in rating objects in imagined social context at encoding. Secondly "Are cultural differences in encoding performance seen in young adult preserved in older adults? And thirdly, "Do cultural differences in encoding, if any, relate to the cultural effects or recognition of encoding context? (Lixia Yang, 2018). For the sample population of this study, the researcher used 72 Canadians of European descent living in Toronto and 72 native Chinese living in Beijing (Lixia Yang, 2018). Experiment required participants to completed two blocks of context memory task, called relational and independent (Lixia Yang, 2018).

In sum, the study discovered that there is a clear cultural difference in encoding of socially meaningful item-context associations. The Chinese participants provided higher rating, took longer to rate, and reported more vivid imagery of encoding contexts than the Canadian counterparts. Also, the results suggest that the previously reported context memory advantage in Chinese may have its origin in the construction of elaborative and meaningful object-context associations at encoding (Lixia Yang, 2018).

Prior theories/research did indicate that there is a cross cultural difference in cognition when it comes to encoding object in imagined social context. In fact, Western and Eastern cultures tend to engage elaborative encoding to form better quality association between objects and imagined social context, relative to the Canadian (Lixia Yang, 2018). They are also more sensitive to the context variance than Canadian participants at encoding. However, these cultural variations tend to be preserved in older adults (Lixia Yang, 2018). Also older adults' context recognition may be more heavily contingent on the effort invested during encoding (Lixia Yang, 2018). However, young adults may be able to retrieve even those object-context pairs encoded with low effort (Lixia Yang, 2018).

Cultural psychologists have found different patterns of thinking and perception in different societies, with some cultures demonstrating a more analytic pattern and others a more holistic pattern. Cultures also differ in their social orientations; independence vs. interdependence. Those that employ independent social orientation tend to emphasize selfdirection, autonomy, and self-expression whereas those that employ interdependent social orientation tend to emphasize harmony, relatedness, and connection. Independently- 
oriented cultures tend to view the self as bounded and separate from others, whereas interdependently-oriented cultures tend to view the self as interconnected with emphasis placed on relationships (Varnum, Grossman, Kitayama \& Nisbett, 2010). In independentlyoriented cultural contexts, happiness is most often experienced as a socially disengaging emotion, whereas in interdependently-oriented cultural contexts, happiness is most often experienced as a socially engaging emotion. Cultures which have an independent social orientation, people are more motivated to enhance their individuality at the expense of others but this is not practiced in interdependently oriented cultures.

Various studies have shown that the covariation between social orientation and cognitive style even occurs outside of North America and East Asia. It is also present in societies that are part of the European cultural tradition. It can therefore be concluded that the cultures which differ in social orientation all differs where cognitive style is concerned. For example, East Europeans and Americans differ along these dimensions. Russians are more interdependent than Americans and they are more holistic in terms of categorization, attribution, visual attention, and reasoning about change (Grossmann, 2009). Similarly, Croats are more interdependent than Americans and they show more holistic patterns of cognition in terms of categorization and visual attention (Varnum, Grossmann, Katunar, Nisbett, \& Kitayama, 2008). Recent evidence additionally suggests that similar differences exist within Europe. Moreover, Russians, who are more interdependent than Germans also show more contextual patterns of visual attention (Medzheritskaya, 2008).

\section{Conclusion}

The problems in the articles used were clearly stated. Despite using different articles, the content was found to be cohesive thrusting showing how differences in culture do in fact have an impact on cognition. There were some minor issues such as the participants used and the lack of literature review based on the questions under investigation. More or additional literature would have allowed readers to thoroughly examine other pieces of research found on the topic as well as how these studies were conducted. Additionally, it would have built up good justification as to why this particular topic was important to research. Most importantly, the overall tone of the articles displayed an unbiased and scientific attitude attesting to its soundness and general credibility. There are several notable cross cultural differences in cognition this was evident in the articles. One line of evidence comes from the fact that priming independence leads to analytic cognition, whereas priming interdependence leads to holistic cognition. When combined, these findings suggest that social orientation does in fact cause cultural differences in cognition.

Additionally, evidence for cultural differences in the specificity of memory for visual information was also found. Americans tend to exhibited greater accuracy in tests of specific memory when compared to East Asians despite performing similarly when general memory was assessed. As a result memory specificity did not seem to affect general recognition of images, which would suggest that difference lies in the quality, but not the quantity, of visual information remembered. It was also found that are clear cultural differences in the encoding of socially or experientially meaningful item-context associations. Chinese participants provided higher rating, took longer to rate, and reported more vivid imagery of encoding contexts when compared to Canadian counterparts. Furthermore, the results suggest that the previously reported context memory advantage in Chinese may have its origin in the construction of elaborative and meaningful objectcontext associations at encoding. Though the evidence shows that differences exist, further research can be conducted to solidify these findings. 


\section{References}

Cherry, K. (2005, December 14). Importance of Cognition in Determining Who We Are. Retrieved from https://www.verywellmind.com/what-is-cognition-2794982

Cognition definition and meaning | Collins English Dictionary. (n.d.). Retrieved from https://www.collinsdictionary.com/dictionary/english/cognition

The Mind in the World: Culture and the Brain. (n.d.). Retrieved from https://www.psychologicalscience.org/observer/the-mind-in-the-world-culture-andthe-brain

Chiao, J. Y., \& Blizinsky, K.D. (2010). Culture-gene coevolution of individualism: Collectivism and the serotonin transporter gene. Proceedings of the Royal Society B: Biological Sciences. 2010;277:529-537

Gentner, D. (1982). Why nouns are learned before verbs: Linguistic relativity versus natural partitioning. In: Kuozaj S, editor. Language development. Language, thought, and culture. Vol. 2. Hillsdale, NJ: Lawrence Erlbaum. pp. 301-334.

Kim, H.S,, Sherman, D. K., Sasaki, J.Y., Xu, J., Chu, T.Q., Ryu, C., et al. (2010). Culture, distress, and oxytocin receptor polymorphism (OXTR) interact to influence emotional support seeking. Proceedings of the National Academy of Sciences of the United States of America.107:15717-15721.

Kuwabara, M., \& Smith, L. B. (2012). Cross-cultural differences in cognitive development: attention to relations and objects. Journal of experimental child psychology, 113(1), 20-35.

Nisbett, R. E., \& Masuda, T. (2003). Culture and point of view. Proceedings of the National Academy of Sciences of the United States of America. 100:11163-11170.

Nisbett, R. E., \& Miyamoto, Y. (2005). The influence of culture: Holistic versus analytic perception. Trends in Cognitive Sciences. 9:467-473. 